\title{
ARTICLE \\ Psilocybin and LSD have no long-lasting effects in an animal model of alcohol relapse
}

\author{
Marcus W. Meinhardt (iD ${ }^{1,2}$, Cansu Güngör ${ }^{1}$, Ivan Skorodumov (D) ${ }^{1}$, Lea J. Mertens ${ }^{2}$ and Rainer Spanagel ${ }^{1}$
}

\begin{abstract}
For most psychiatric disorders, including alcohol use disorder (AUD), approved pharmacological treatments are limited in their effectiveness, and new drugs that can easily be translated into the clinic are needed. Currently, great hope lies in the potential of psychedelics to effectively treat AUD. The primary hypothesis is that a single session of psychedelic-guided psychotherapy can restore normal brain function in AUD individuals and thereby reduce the risk of relapse in the long run. Here we applied three different treatment schedules with psilocybin/LSD in order to investigate relapse-like drinking in the alcohol deprivation effect (ADE) model. In contrast to the primary hypothesis, psychedelics had no long-lasting effects on the ADE in male and female rats, neither when administered in a high dosage regime that is comparable to the one used in clinical studies, nor in a chronic microdosing scheme. Only sub-chronic treatment with psilocybin produced a short-lasting anti-relapse effect. However, it is not a translatable treatment option to give psychedelics sub-chronically for relapse prevention. In conclusion, our results in the ADE model do not support the hypothesis that microdosing or high doses of psychedelic reduce relapse behavior. This conclusion has to be confirmed by applying other animal models of AUD. It could also well be that animal models of AUD might be unable to fully capture the therapeutic potential of psychedelic drugs and that only future large-scale clinical trials will be able to demonstrate the efficacy of psychedelics as a new treatment option for AUD.
\end{abstract}

Neuropsychopharmacology (2020) 45:1316-1322; https://doi.org/10.1038/s41386-020-0694-z

\section{INTRODUCTION}

"High hopes-Psychedelic drugs fell from grace in the 1960s. Now, scientists are rediscovering them as potential treatments for a range of psychiatric disorders" was the recent title of a Feature Article in Science [1]. Indeed, psychedelics are one of the hottest topics in psychiatry with lots of hope; but do we already have enough scientific evidence that would justify psychedelics as a treatment option? Despite the hype and launch of several clinical trials, so far surprisingly little research especially in animal models of psychiatric disorders has been conducted [2-5]. Here we used a well-established animal model of alcohol relapse to test the hypothesis whether the psychedelic drugs psilocybin and LSD will exhibit long-lasting anti-relapse properties in alcohol use disorder (AUD) individuals when given at high doses. In addition, we studied the effect of microdosing on relapse behavior. A microdose is considered a sub-hallucinogenic dose of a psychedelic compound that may enhance cognitive performance and mental health $[6,7]$. In a recent anonymous online survey, microdosers were significantly less likely to report a history of substance use disorders when compared with non-microdosers [8]. These findings provide promising initial evidence that warrants controlled experimental research to directly test the potential benefits and risks of microdosing in AUD or substance use disorders.

AUD is a major problem and disease burden for affected patients, but also society due to its immense economic costs. The 12-month prevalence rate of AUD, as defined by the Diagnostic and Statistical Manual of Mental Disorders (DSM)-5, is around $14 \%$. Hence AUD is highly prevalent, physically and psychologically disabling, and often occurs comorbid to other psychopathologies and somatic conditions [9]. In fact, excessive alcohol drinking puts people at risk for various other diseases, including neuropsychiatric disorders, and is linked to cancer, cardiovascular diseases, and liver cirrhosis [10].

Currently, disulfiram, naltrexone, and acamprosate have been approved by most regulatory bodies for the treatment of AUD and are available in most countries across the globe [11]. Nalmefene has also recently been approved for the reduction of alcohol consumption. However, these pharmacological treatments for AUD are limited in their effectiveness [12], and new drugs are warranted. Moreover, drug development for AUD is highly challenging due to methodological issues of alcohol pharmacotherapy trials $[12,13]$.

Because of the scarcity in effective pharmacological interventions for AUD and relapse prevention, interest in psychedelic drugs, including the ergoline lysergic acid diethylamide (LSD) and the tryptamine Psilocybin (3-[2-(dimethylamino) ethyl]-1H-indol-4yl] dihydrogen phosphate), as a new treatment option has regrown in the past decade $[4,14,15]$.

A meta-analysis of six randomized controlled trials (RCT) conducted from 1966 to 1970 (i.e., before the Schedule I placing by the UN Convention on Drugs) on the efficacy of LSD in the

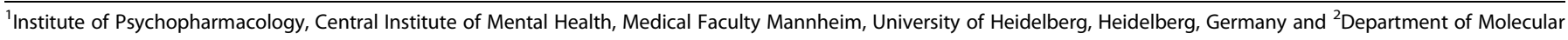
Neuroimaging, Central Institute of Mental Health, Medical Faculty Mannheim, University of Heidelberg, Heidelberg, Germany

Correspondence: Marcus W. Meinhardt (marcus.meinhardt@zi-mannheim.de)

These authors contributed equally: Marcus W. Meinhardt, Cansu Güngör

Received: 10 February 2020 Revised: 21 April 2020 Accepted: 23 April 2020

Published online: 5 May 2020 
Psilocybin and LSD have no long-lasting effects in an animal model of... MW Meinhardt et al.

context of different AUD treatment programs revealed beneficial effects on alcohol misuse of a single LSD dose [16]. While modern clinical trials with LSD are lacking, Bogenschütz et al. [17] recently conducted a proof-of-concept clinical trial for psilocybin in AUD, in which they administered two high doses $(0.3 \mathrm{mg} / \mathrm{kg}$ and 0.3 or 0.4 $\mathrm{mg} / \mathrm{kg}$ ) of psilocybin as part of a 12-week manualized psychotherapeutic intervention in 10 patients with AUD. The results show some preliminary efficacy in decreasing drinking behavior in weeks 5-12 relative to baseline and relative to weeks $1-4$ (i.e., before the first psilocybin dose).

While these preliminary results of clinical trials on the beneficial therapeutic effects of psilocybin and LSD are promising, wellcontrolled studies are missing and there is a lack of understanding of the mechanisms (psychological and neurobiological) underlying the putative anti-relapse effects. However, comprehension of those mechanisms is essential in order to optimize safety and efficacy when administering LSD or psilocybin. Animal models provide a unique opportunity to study those neurobiological effects and aid to address the ongoing debate whether the therapeutic effects of psychedelic drugs are driven by neurobiological, psychological changes, or both.

The present study aimed to investigate the effects of LSD and psilocybin in a rat model of alcohol relapse, namely the alcohol deprivation effect (ADE) model in long-term alcohol drinking Wistar rats [18]. The ADE model is regarded as a DSM-based animal model of relapse as it involves compulsive drinking, wanting (craving), and loss of control in a relapse situation [18, 19]. In the ADE model, renewed access to alcohol solutions after a period of deprivation commonly leads to a pronounced (although temporary) increase in voluntary alcohol intake (i.e., the ADE) in animals. After repeated deprivation phases, the animals show an increased demand for the drug that resembles a relapse in patients with AUD $[20,21]$. Previous studies provided pharmacological and predictive validation of the model because an attenuation of the ADE could be demonstrated through repeated sub-acute treatment with approved pharmacological treatment agents of AUD (e.g., acamprosate, naltrexone) [21, 22].

Several human studies showed that single hallucinogenic doses of psychedelics can produce long-lasting changes in mood and behavior [4, 23, 24]. In line with this, experiments in rodents suggest that a single hallucinogenic dose of psychedelics can change the brain structure and behavior long after the drug has been cleared from the body $[25,26]$. The ADE model has been proven to be sensitive to long-lasting treatment effects $[27,28]$. Therefore, the current study tested the hypothesis that an acute high dose psychedelic intervention-as used in clinical settingswill have long-lasing beneficial effects on alcohol relapse. In addition, we tested the hypothesis that microdosing of a psychedelic drug will reduce relapse behavior in the ADE model. As preclinical and clinical evidence suggests that sex influences disease trajectories and interventions in AUD patients, male and female rats were studied in direct comparison [29].

\section{MATERIALS AND METHODS}

\section{Animals}

Ninety-one 2-month-old male and female Wistar rats (from our own breeding colony at the Central Institute of Mental Health, Mannheim, Germany) were used. All animals were housed individually in standard rat cages under a $12 \mathrm{~h}$ artificial light/dark cycle (lights on at 6:00 a.m.). Room temperature was kept constant (temperature: $22 \pm 1{ }^{\circ} \mathrm{C}$, humidity: $55 \pm 5 \%$ ). Standard laboratory rat food and water were provided ad libitum throughout the experimental period. Body weights were measured weekly. All experiments were approved by the institutional Committees on Animal Care and Use, and by the Regierungspräsidium Karlsruhe, and were performed in accordance with the European and German national guidelines.
Drugs

See Supplementary Information for details.

Long-term voluntary alcohol consumption with repeated deprivation phases

To model relapse-like drinking in rats we applied the ADE model as previously published [27, 30]. After 2 weeks of habituation to the animal room, rats were given ad libitum access to tap water and to 5,10 , and $20 \%$ ethanol solutions ( $/ \mathrm{v}$ ) as well. Spillage and evaporation were minimized by the use of special bottle caps. With this procedure the ethanol concentration remains constant for at least 1 week [31]. The positions of bottles were changed weekly. The first deprivation period was introduced after 8 weeks of continuous alcohol availability. After a deprivation period (between 3 and 4 weeks), rats were given access to alcohol again and two more deprivation periods were introduced in a random manner, i.e., the duration of following drinking and deprivation phases was irregular, i.e., $\sim 6 \pm 1$ week and $4 \pm 1$ week, respectively in order to prevent adaptive behavioral mechanisms $[27,30]$. The long-term voluntary alcohol drinking procedure including all deprivation phases lasted a total of 9 months.

In order to study the effects of different psychedelic drugs in comparison to vehicle, rats were divided into three groups $(n=8-10)$ in such way that the mean baseline total alcohol intake was approximately the same in each group. Baseline drinking was measured daily for 1 week. After the last day of baseline measurement, the alcohol bottles were removed from the cages, leaving the animals with free access to food and water for the complete deprivation period.

In the current study we performed three experiments with different drug administration schemes before reintroducing the alcohol bottles and assessment of the ADE (Fig. 1). The chosen drug administration schemes were (i) a repeated dosing scheme (i.e., five doses across three days, starting Monday night, followed by administration twice daily on Tuesday and Wednesday) based on an established paradigm used in our previous ADE studies to test alcohol relapse behavior [27, 32, 33]. (ii) Two high dose applications 1 week apart as to resemble the administration scheme of human clinical trials [e.g., [17]]. (iii) A 4-week microdosing regime with two doses a week [34]. The rationale for these different schemes was to first use our well established application scheme in rats to test pharmacologically active drugs in the ADE model (Experiment 1). Next we used translatable treatment schemes to test our hypothesis that a prophylactic psychedelic treatment has long-lasting effects on relapse behavior using a prophylactic treatment scheme, either with two high doses (experiment 2) or eight small doses (experiment 3). Details of all three experiments are provides below.

Experiment 1: The effect of psilocybin on alcohol relapse Following a period of abstinence, each animal was subjected to a total of five intraperitoneal (i.p.) injections starting at 7 p.m. in the evening before the reintroduction of the alcohol bottles, followed by four applications in $12 \mathrm{~h}$ intervals of either vehicle or a moderate dose of psilocybin $(1 \mathrm{mg} / \mathrm{kg})$. This dose has been identified as the minimal dose to induce behavioral effects in rats [35] and Mark Geyer and Franz X. Vollenweider personal communications]. The alcohol bottles were reintroduced after the second injection (at $~ 9$ a.m.) and the occurrence of an ADE was determined. Total ethanol ( $\mathrm{g} / \mathrm{kg}$ of body weight/day) and water intake $(\mathrm{ml} / \mathrm{kg}$ of body weight/day) were measured daily at $\sim 9$ a.m. for the subsequent week. In order to test for persistent treatment effects, both ethanol and water intake were measured for three more weeks. Each rat's body weight was recorded $24 \mathrm{~h}$ before the first injection and $12 \mathrm{~h}$ after the last injection. In addition, home cage locomotor activity was monitored by use of an infrared sensor connected to a recording and data storing system (Mouse-E-Motion, Infra-e-motion, Henstedt-Ulzburg, 
A: Experiment 1: 5x moderate dose, every 12h

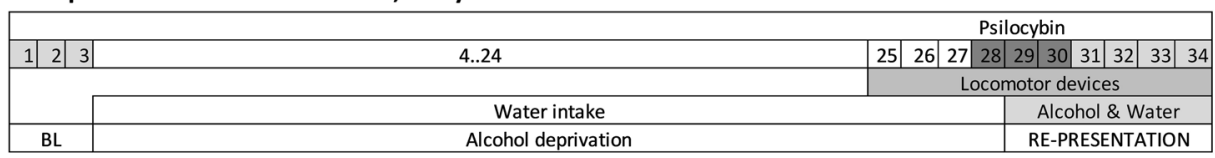

B: Experiment 2: 2x high doses, 7days apart

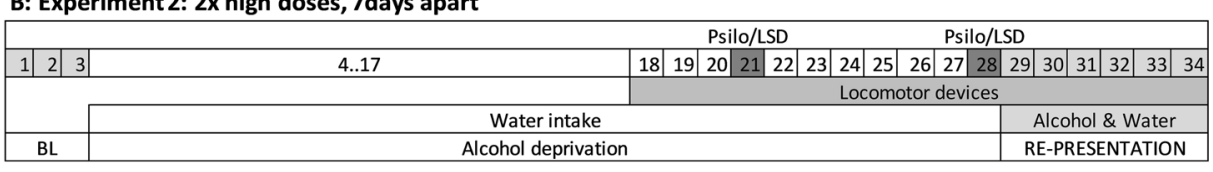

C: Experiment 3: 8x microdoses within 4weeks

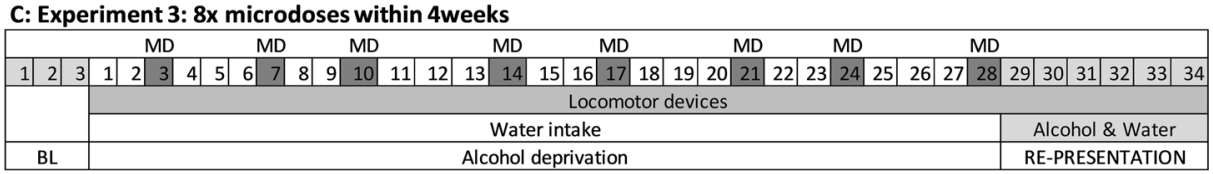

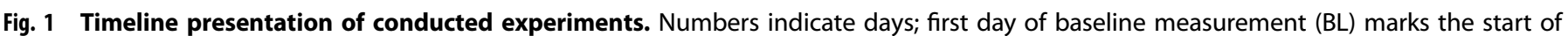

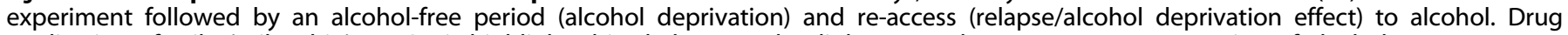
application of Psilo (Psilocybin) or LSD is highlighted in dark gray color; light gray colors represent consumption of alcohol.

Germany). A Mouse-E-Motion device was placed above each cage $(30 \mathrm{~cm}$ from the bottom) so that the rat could be detected at any position inside the cage. The device was sampling every second, whether the rat was moving or not. The sensor could detect body movement of the rat of at least $1.5 \mathrm{~cm}$ from one sample point to the successive one. The data measured by each Mouse-E-Motion device were downloaded into a personal computer and processed with Microsoft Excel.

Experiment 2: The long-term effects of psilocybin and LSD with medium to high doses on alcohol relapse

In order to develop a robust treatment approach that can be translated to the clinical situation, we asked whether pretreatment with either psilocybin or LSD is able to reduce alcohol relapse in rats on the long run. We adapted the treatment schedule from a recent proof-of-concept clinical study in patients with AUD [17] and administered twice (7 days apart) two different doses (i.p. injection) of psilocybin (group 1: $2.5 \mathrm{mg} /$ $\mathrm{kg}$ twice; group 2: $10 \mathrm{mg} / \mathrm{kg}$ twice) or LSD (group 1: $0.08 \mathrm{mg} / \mathrm{kg}$ twice; group 2: $0.32 \mathrm{mg} / \mathrm{kg}$ twice) during the deprivation period and investigated their effect on ADE. Doses were selected based on previous publications on rodent psilocybin [36] and LSD studies [37] and on advice by Mark Geyer and Franz X. Vollenweider. The first injection was scheduled 7 days before, and the second injection the evening before ADE ( $15 \mathrm{~h}$ before $A D E$ in order to avoid any locomotion impairment of the high doses). Food and water intake as well as locomotion was measured starting 3 days before the first injection. The alcohol bottles were reintroduced the morning after the second injection (at $\sim 9$ a.m.) and the occurrence of an ADE was determined. As reported for experiment 1, total ethanol $(\mathrm{g} / \mathrm{kg}$ of body weight/day) and water intake ( $\mathrm{ml} / \mathrm{kg}$ of body weight/ day) were measured daily at $\sim 9$ a.m. for the subsequent week. In order to test for persistent treatment effects, both ethanol and water intake were measured for three more weeks. Each rat's body weight was recorded $24 \mathrm{~h}$ before each of the two injections. In addition, home cage locomotor activity was measured by the E-motion system.

Experiment 3: The effect of chronic intermittent microdosing of psilocybin during abstinence on subsequent relapse-like drinking behavior

Contrasting psychedelic pre-treatment with single or very few high doses, recent rodent literature in the field of depression provides evidence for beneficial effects on mood and anxiety after chronic intermittent microdosing of the psychedelic tryptamine $\mathrm{N}$, $\mathrm{N}$-dimethyltryptamine [34]. A microdose is considered a sub- hallucinogenic dose of a psychedelic compound and is rapidly gaining popularity in humans. Despite the prevalence of microdosing, the field lacks peer-reviewed studies that investigate the potential benefits and risks of microdosing, especially for substance use disorders. In addition, there is no well-established definition of a "microdose". In humans, typically $1 / 10$ of a hallucinogenic dose is considered as microdose [38]. Since $1 \mathrm{mg} /$ $\mathrm{kg}$ psilocybin has been generally used in rats to investigate behavioral effects [35, 36], we opted to administer psilocybin at $0.1 \mathrm{mg} / \mathrm{kg}$ and defined it as a microdose in rats. This dose has also been shown to produce $\sim 10-20 \%$ drug appropriate responding in rats trained to discriminate psilocybin with other hallucinogens and can therefore be seen as sub-hallucinogenic threshold dose [37].

Thus, within the 4-week deprivation period, we applied in total eight injections (i.p.) of either vehicle or psilocybin $(0.1 \mathrm{mg} / \mathrm{kg})$; with two injections per week (applications on Tuesdays and Fridays, $\sim 5$ p.m.) to male and female rats. The alcohol bottles were reintroduced the morning after the last injection (at $\sim 9$ a.m.) and the occurrence of an ADE was determined. As reported for experiment 1 and 2, total ethanol ( $\mathrm{g} / \mathrm{kg}$ of body weight/day) and water intake $(\mathrm{ml} / \mathrm{kg}$ of body weight/day) were measured daily at $\sim 9$ a.m. for the subsequent week. In order to test for persistent treatment effects, both ethanol and water intake were measured for three more weeks. Each rat's body weight was recorded weekly within the microdosing period. In addition, home cage locomotor activity was measured by the E-motion system.

\section{Statistics}

Data derived from home-cage drinking (total alcohol intake and water intake) and home-cage locomotor activity were analyzed using a two-way repeated measures Analysis of Covariance (ANCOVA), with treatment as the between-subject factor and day/week as the within-subject factor. Data from males and females were pooled and sex was added as covariate. Since male and female rats differ significantly in their drinking behavior, baseline alcohol and water intake outcome variables as well as locomotion were centered to the respective group mean. To visualize differences between sexes, individual subgroup graphs for each sex are provided in each experiment. However, statistics were exclusively applied to the pooled group containing males and females. Locomotion, alcohol and water intake on posttreatment days were expressed as the percentage relative to baseline drinking. Whenever significant differences were found, posthoc Student Newman Keuls tests were performed. All statistical analyses were conducted with Statistica 13.3 (Statsoft, Hamburg, Germany). 

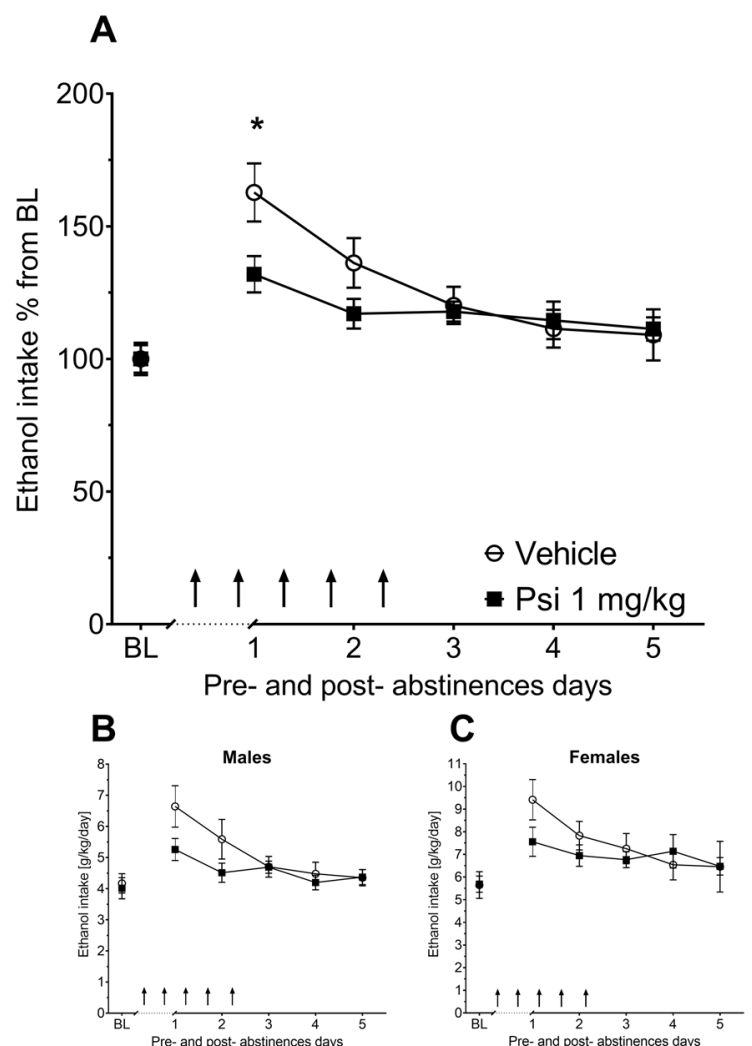

Fig. 2 Effects of acute psilocybin on relapse-like drinking. a Intake of ethanol in percent to mean baseline values for all animals before and after a deprivation period of 4 weeks. The last 3 days measurements of ethanol intake is given as baseline drinking - "BL". Arrows indicate the administration of either vehicle, or $1 \mathrm{mg} /$ kg of Psilocybin (Psi) ( $n=8-10$ per treatment condition and sex). b, $\mathbf{c}$ Intake of total ethanol (calculated in $\mathrm{g}$ of pure alcohol per $\mathrm{kg}$ of body weight per day) representative graphs for each sex. Data are presented as means \pm SEM. * significant differences from the vehicle control group, $p<0.05$.

\section{RESULTS}

Experiment 1: The effect of psilocybin on alcohol relapse

At first, we investigated whether psilocybin is capable to act as anti-relapse treatment using an established treatment schedule for the ADE measurements. Opposed to males, female animals have the ability of maintaining stable body weights (within 10\%) over time and since alcohol intake is calculated based on each animal's body weight, female rats consumed significantly more alcohol than male rats. Absolute mean baseline values for alcohol intake $[\mathrm{g} / \mathrm{kg} /$ day $\pm \mathrm{SEM}]$ were: vehicle group males: $4.17 \pm 0.31$, psilocybin group males: $4.02 \pm 0.33$; vehicle group females: $5.65 \pm$ 0.59 , psilocybin group females: $5.69 \pm 0.35$ ). In order to pool the analysis of males and females, baseline alcohol and water intake outcome variables as well as locomotion were centered to the respective group mean and displayed in panels "a" of all figures. To visualize differences between sexes, individual graphs (panels " $b$ " and " $c$ ") for each sex are provided for each experiment. The vehicle-treated group showed the expected increase in alcohol consumption, indicating occurrence of an ADE (Fig. 2a-c). A twoway ANCOVA for repeated measures revealed a significant increase in alcohol intake after a deprivation phase in all animal groups as compared with basal drinking [factor day $F(5,165)=2.59$, $p<0.027]$. This increase was mainly attributable to the vehicletreated group, whereas psilocybin treatment reduced the expression of ADE (Fig. 2a). Alcohol intake was significantly reduced in psilocybin-treated rats as confirmed by the significant treatment group $\times$ day interaction effect $[F(5,165)=3.89, p<0.002]$. Sex as covariate was not found to be statistically significant $[F(1,33)=$ $0.84, p<0.36]$. Water intake (Supplementary Fig. 1) was not different in psilocybin-treated animals compared with controls during treatment days, suggesting that the effect of treatment was selective for alcohol (factor treatment group $\times$ day interaction effect $[F(5,165)=0.42, p=0.83)$. Home-cage locomotor activity measurements for the ADE experiment were analyzed in $30 \mathrm{~min}$ blocks over the entire experiment. Overall, there was a general reduction during the first three days of $A D E$ in home-cage activity seen in all animal groups, which is regularly observed in ADE experiments and was likely caused by alcohol intoxication. In line with this conclusion, a repeated measurement ANOVA revealed no significant difference in activity of rats treated with psilocybin, when compared with the vehicle-treated group [factor treatment group $\times$ day interaction effect: $F(8,152)=0.67, p=0.71$ ] (Supplementary Fig. 2). In a detailed post-hoc sub-group analysis for males and females, we observed that locomotor activity was reduced for $2 \mathrm{~h}$ following psilocybin administration exclusively in males. No such difference was observed in female rats.

Experiment 2: The long-term effects of psilocybin and LSD with medium to high doses on alcohol relapse

As described, we adapted the treatment schedule from the clinical trial by Bogenschutz et al. [17] and administered twice (seven days apart) two different high doses of psilocybin $(2.5$ or $10 \mathrm{mg} / \mathrm{kg}$ ) or LSD $(0.08$ or $0.32 \mathrm{mg} / \mathrm{kg}$ ) during abstinence and investigated the effect of such a pre-/prophylactic treatment on ADE. All treated groups showed the expected increase in alcohol consumption, indicating occurrence of an ADE (Figs. 3, 4). A two-way ANCOVA for repeated measures showed a significant increase in alcohol intake after the deprivation phase in all animal groups as compared to basal drinking [factor day for psilocybin: $F(5,275)=$ 14.42, $p<0.0001$; LSD: $F(5,250)=11.18, p<0.0001]$. However, there was no difference of either psilocybin or LSD treatment for the tested doses on ADE (Figs. 3, 4). Measurements on potential side effects of the treatment, (total fluid intake and locomotion) also showed no differences between groups (data not shown).

Experiment 3: The effect of chronic intermittent microdosing of psilocybin during abstinence on subsequent relapse-like drinking behavior

During the microdosing regime, rats received in total eight injections i.p. of either vehicle or psilocybin $(0.1 \mathrm{mg} / \mathrm{kg})$ within the 4-week abstinence period, two injections per week (applications on Tuesdays and Fridays). The alcohol bottles were reintroduced $16 \mathrm{~h}$ after the last application. Once again, all groups of rats showed a significant increase in alcohol consumption, indicating occurrence of an ADE (Fig. 5). A two-way ANCOVA for repeated measures showed a significant increase in alcohol intake after the deprivation phase in all animal groups as compared to basal drinking [factor day: $F(5,165)=5.64, p<0.0001$ ]. However, similar to the high-dose application schedule, no difference of psilocybin treatment compared to vehicle on ADE was observed (Fig. 5a-c). Measurements on potential side effects of the treatment, (total fluid intake and locomotion) also showed no differences between groups (data not shown).

\section{DISCUSSION}

In the present study we applied three different treatment schedules with psychedelics in order to investigate the effect of psilocybin and LSD on relapse-like drinking in the ADE rat model. The chosen drug administration schemes were (i) a repeated subchronic dosing scheme (i.e., five doses across 3 days) based on a well-established paradigm used in our previous ADE studies to test relapse behavior [e.g., [27, 33]. (ii) two high dose applications 

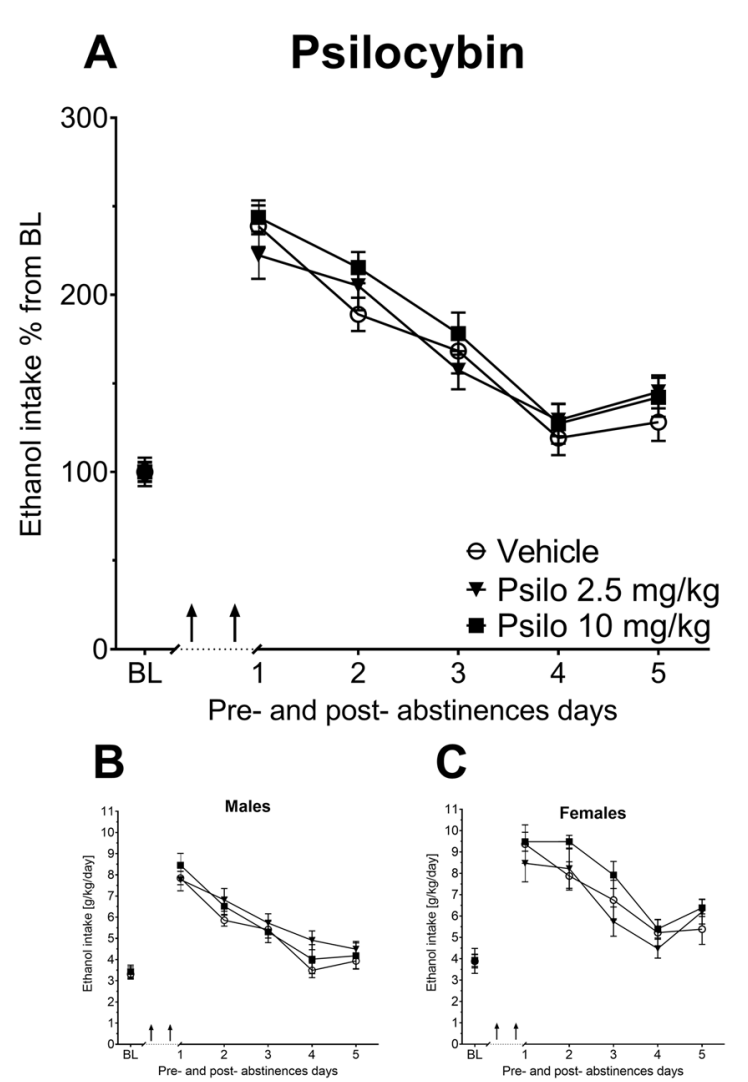

Fig. 3 The long-term effects of psilocybin with medium to high doses on alcohol relapse. a Intake of ethanol in percent to mean baseline values for all animals before and after a deprivation period of 4 weeks. The last 3 days measurements of ethanol intake is given as baseline drinking - "BL". Arrows indicate the administration of either vehicle or 2.5 vs. $10 \mathrm{mg} / \mathrm{kg}$ of Psilocybin (Psi) $(n=9-10$ per treatment condition and sex). b, c Intake of total ethanol (calculated in $\mathrm{g}$ of pure alcohol per $\mathrm{kg}$ of body weight per day) representative graphs for each sex. Data are presented as means \pm SEM.

one week apart as to resemble the administration scheme of a published human clinical study [17], and (iii) a 4-week microdosing regime with two doses per week. In contrast to our a priori hypothesis, psychedelics had no long-lasting effects on the ADE in rats neither when administered in a dosage regime that is comparable to the one used in clinical trials and which showed preliminary efficacy in humans with AUD [17], nor in the chronic microdosing scheme. Only sub-chronic treatment with psilocybin, which is a standard application scheme in rats to test pharmacologically active drugs in the ADE model, produced a short-lasting anti-relapse effect. However, it is clearly not a translatable treatment option to give psychedelics subchronically or chronically for relapse prevention.

Animal models allow the specific testing of pure pharmacological effects of psychedelic drugs since no psychological support can be provided and no expectancy effects exist. Experiments in rodents suggests that a single hallucinogenic dose of psychedelics can change the brain structure and behavior long after the drug has been cleared from the body $[25,26]$. Moreover, a preclinical study published by Alper et al. [39] showed reductions in both ethanol consumption and preference in a group of mice treated with a single LSD dose of $0.05 \mathrm{mg} / \mathrm{kg}$. Although the magnitude of effect on ethanol consumption in this study was small, with a group mean reduction of $18 \%$, it was sustained across a time interval of 46 days. At the moment we cannot provide any explanation for the difference in results of the study by Alper et al. [39] and the here presented results. We used slightly higher doses
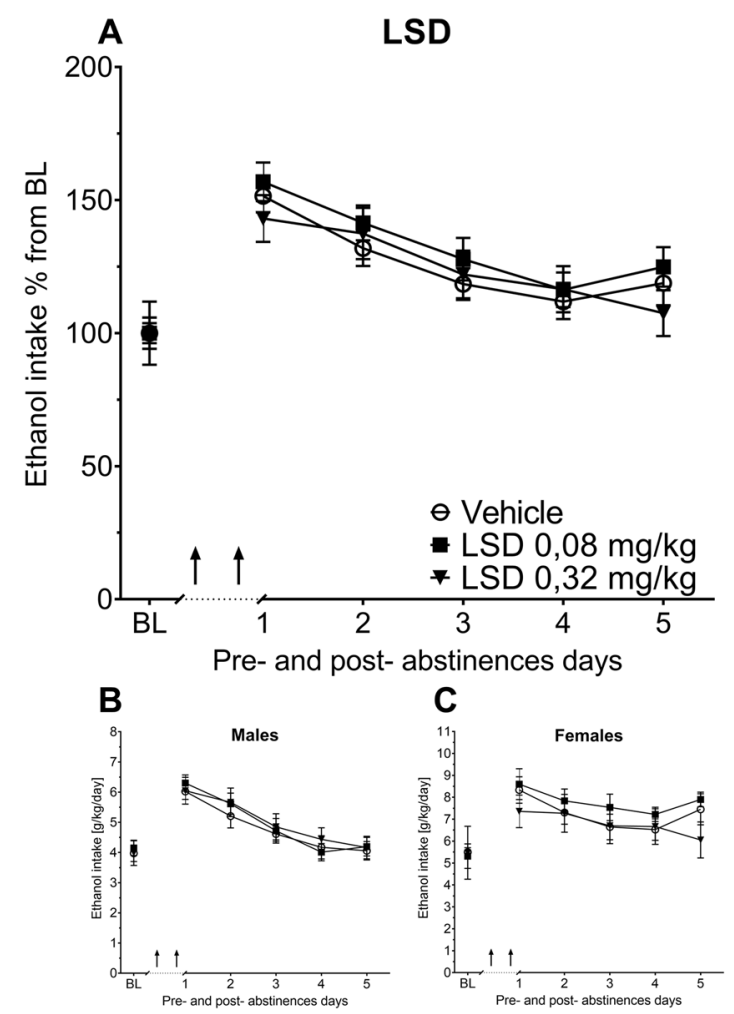

Fig. 4 The long-term effects of LSD with medium to high doses on alcohol relapse. a Intake of ethanol in percent to mean baseline values for all animals before and after a deprivation period of 4 weeks. The last 3 days measurements of ethanol intake is given as baseline drinking-“'BL'". Ar rows indicate the administration of either vehicle or 0.08 vs. $0.32 \mathrm{mg} / \mathrm{kg}$ of LSD $(n=9$ per treatment condition and sex). b, c Intake of total ethanol (calculated in $\mathrm{g}$ of pure alcohol per kg of body weight per day) representative graphs for each sex. Data are presented as means \pm SEM.

of LSD ( 0.08 and $0.32 \mathrm{mg} / \mathrm{kg}$ ) but could not see any effect even not at the first day of ADE drinking which started $12 \mathrm{~h}$ post treatment. Moreover, psychedelic pretreatment with microdosing, a scheme that has growing popularity in recent years $[6-8,40,41]$, but lacks peer-reviewed and controlled studies that investigate the potential benefits and risks of microdosing, especially for substance use disorders, did not affect relapse-like drinking in rats. Other rodent studies also generated mixed results with a microdosing scheme on mood and anxiety [34, 42].

Numerous previous studies on the serotonin (5-HT) system in the ADE model support the notion that targeting the serotonergic system might be beneficial for relapse prevention [43]. In addition, psilocybin has been shown to activate $5-\mathrm{HT} 2 \mathrm{~A}$ receptors in the medial prefrontal cortex (mPFC), which leads to increases in the firing rate of 5-HT neurons in the dorsal raphe and of dopamine neurons in the ventral tegmental area. The activation results in an augmented release of $5-\mathrm{HT}$ in the $\operatorname{mPFC}[44,45]$ and of dopamine in mesocortical areas [46] in animals. In humans, psilocybin also increases striatal dopamine concentrations, which was found to be correlated with euphoria [47]. Thus, the observed short-lasting effect on ADE in experiment 1 could be attributed to increased mesocotrical dopamine through acute psilocybin administration, acting as rewarding component and thus reducing the ADE. However, this interpretation is speculative and has to be tested in further experiments focusing on molecular mechanisms.

Relapse prevention through prophylactic treatments as tested in experiment 2 and 3 with either pretreatment with high does or microdosing, likely requires molecular machineries involved in 

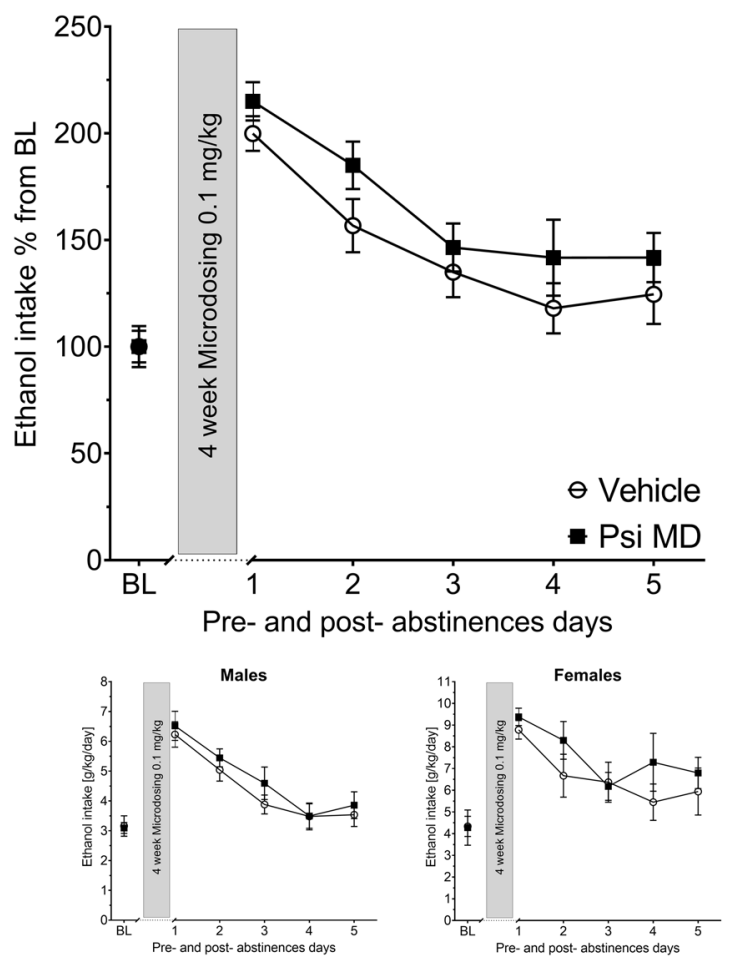

Fig. 5 Effects of psilocybin microdosing on relapse-like drinking. Intake of total ethanol (calculated in $\mathrm{g}$ of pure alcohol per $\mathrm{kg}$ of body weight per day) before and after a deprivation period of 4 weeks. The last 3 days measurements of ethanol intake is given as baseline drinking-"BL". The gray bars indicate the 4 weeks of microdosing (MD) administration of either vehicle or psilocybin (Psi) $0.1 \mathrm{mg} / \mathrm{kg}$ ( $n=9$ per treatment condition and sex). Data are presented as means \pm SEM.

neuronal plasticity leading to lasting changes in network activity and connectivity. LSD has been shown to mediate its effects via immediately early gene expression, predominantly within the prefrontal cortex [48]. Among the strongest induced genes is the early growth response factor 2 (Egr2), which is a critical regulator for long-term, activity-dependent adaptive responses of neurons such as in processes of neuronal plasticity in response to synaptic activation and the formation of long-term memories for newly learned events [49]. However, our previous studies showed that Egr2 is highly downregulated within the mPFC in alcoholdependent rats [50]; therefore failure to activate Egr2 of LSD in alcohol-dependent rats could explain the lacking effect observed in experiment 2. Since this study focusses on a behavioral pharmacology set of result, this conclusion is again speculative and needs to be tested in other experiments.

Despite these observed negative results with psychedelic pretreatment, the positive finding of reduced ADE with acute psilocybin treatment suggests that the efficacy of psilocybin for the treatment of AUD reported in a preliminary clinical study [17] may also involve a biologically mediated effect and is not solely due to the therapeutic effects of the subjective psychedelic experience. In general, the settings in which psychedelics have previously been administered as treatment for AUD vary. A RCT showed positive results in which patients were strapped to a bed in a hospital room [51], also suggesting that the effect of psychedelics on alcohol misuse may have involved a pharmacological component distinct from psychotherapeutic care and setting.

In summary, the classic psychedelics LSD and psilocybin had no effect on relapse behavior in the ADE model when given at a supposedly high dose. Microdosing with psilocybin did also not affect relapse-like drinking behavior in this animal model. To further support these negative findings more preclinical studies are warranted that use other dosing regimes and other animal model of AUD, e.g., the post-dependent model [52] or alcohol-preferring rat lines [e.g., [53]]. From the present results we can only draw limited conclusion for the human situation especially since animal model cannot capture the different aspects of psychedelic-assisted psychotherapy.

\section{FUNDING AND DISCLOSURE}

Financial support for this work was provided by the Bundesministerium für Bildung und Forschung (BMBF) funded ERA-NET program: Psi-Alc (FKZ: 01EW1908), the BMBFfunded SysMedSUDs consortium (FKZ: 01ZX1909A), and the Deutsche Forschungsgemeinschaft (DFG, German Research Foundation)-Project-ID 402170461-TRR 265 [54]. The authors declare no competing financial interests.

\section{AUTHOR CONTRIBUTIONS}

MWM, CG, and RS designed research; MWM, CG, IS, and LJM performed research; MWM, CG, IS, and LM analyzed data; MWM, IS, LJM, and RS wrote the paper.

\section{ADDITIONAL INFORMATION}

Supplementary Information accompanies this paper at (https://doi.org/10.1038/ s41386-020-0694-z)

Publisher's note Springer Nature remains neutral with regard to jurisdictional claims in published maps and institutional affiliations.

\section{REFERENCES}

1. Kupferschmidt K. High hopes. Science. 2014;345:18-23.

2. dos Santos RG, Hallak JEC. Therapeutic use of serotoninergic hallucinogens: a review of the evidence and of the biological and psychological mechanisms. Neurosci Biobehav Rev. 2020;108:423-34.

3. Liechti ME. Modern clinical research on LSD. Neuropsychopharmacology 2017;42:2114-27.

4. Carhart-Harris RL, Goodwin GM. The therapeutic potential of psychedelic drugs: past, present, and future. Neuropsychopharmacology 2017;42:2105-13.

5. Hibicke M, Landry AN, Kramer HM, Talman ZK, Nichols CD. Psychedelics, but not ketamine, produce persistent antidepressant-like effects in a rodent experimental system for the study of depression. ACS Appl Mater Interfaces. 2020. https://doi. org/10.1021/acschemneuro.9b00493.

6. Polito V, Stevenson RJ. A systematic study of microdosing psychedelics. PLoS ONE. 2019;14:e0211023.

7. Anderson T, Petranker R, Rosenbaum D, Weissman CR, Dinh-Williams LA, Hui $K$ et al. Microdosing psychedelics: personality, mental health, and creativity differences in microdosers. Psychopharmacology. 2019;236:731-40.

8. Rosenbaum D, Weissman C, Anderson T, Petranker R, Dinh-Williams LA, Hui K, et al. Microdosing psychedelics: demographics, practices, and psychiatric comorbidities. J Psychopharmacol. 2020:269881120908004. https://doi.org/ $10.1177 / 0269881120908004$ [Epub ahead of print].

9. Grant BF, Chou SP, Saha TD, Pickering RP, Kerridge BT, Ruan WJ, et al. Prevalence of 12-month alcohol use, high-risk drinking, and DSM-IV alcohol use disorder in the United States, 2001-2 to 2012-3: results from the National Epidemiologic Survey on Alcohol and Related Conditions. JAMA Psychiatry. 2017;74:911-23.

10. Rehm J, Mathers C, Popova S, Thavorncharoensap M, Teerawattananon Y, Patra J. Global burden of disease and injury and economic cost attributable to alcohol use and alcohol-use disorders. Lancet. 2009;373:2223-33.

11. Spanagel R, Vengeliene V. New pharmacological treatment strategies for relapse prevention. Curr Top Behav Neurosci. 2012;13:583-609.

12. Fertig J, Leggio L, Litten RZ, Falk DE, Ryan ML. Advances in pharmacotherapy development: human clinical studies. Handbook of experimental pharmacology. Springer Nature, Switzerland. 2018;248:579-613.

13. Yardley MM, Ray LA. Medications development for the treatment of alcohol use disorder: insights into the predictive value of animal and human laboratory models. Addict Biol. 2017;22:581-615.

14. Bogenschutz MP. Studying the effects of classic hallucinogens in the treatment of alcoholism: rationale, methodology, and current research with psilocybin. Curr Drug Abus Rev. 2013;6:17-29.

15. Rucker JJH, lliff J, Nutt DJ. Psychiatry \& the psychedelic drugs. Past, present \& future. Neuropharmacology. 2018;142:200-18.

16. Krebs TS, Johansen PØr. Lysergic acid diethylamide (LSD) for alcoholism: metaanalysis of randomized controlled trials. J Psychopharmacol. 2012;26:994-1002. 
17. Bogenschutz MP, Forcehimes AA, Pommy JA, Wilcox CE, Barbosa P, Strassman RJ. Psilocybin-assisted treatment for alcohol dependence: a proof-of-concept study. J Psychopharmacol. 2015;29:289-99.

18. Spanagel R. Animal models of addiction. Dialogues Clin Neurosci. 2017; 19:247-58.

19. Vengeliene V, Bilbao A, Spanagel R. The alcohol deprivation effect model for studying relapse behavior: a comparison between rats and mice. Alcohol. 2014;48:313-20.

20. Vengeliene V, Celerier E, Chaskiel L, Penzo F, Spanagel R. Compulsive alcohol drinking in rodents. Addict Biol. 2009;14:384-96.

21. Hölter SM, Spanagel R. Effects of opiate antagonist treatment on the alcohol deprivation effect in long-term ethanol-experienced rats. Psychopharmacology. 1999;145:360-9.

22. Spanagel R, Hölter SM, Allingham K, Landgraf R, Zieglgänsberger W. Acamprosate and alcohol: I. Effects on alcohol intake following alcohol deprivation in the rat. Eur J Pharm. 1996;305:39-44.

23. Griffiths RR, Richards WA, McCann U, Jesse R. Psilocybin can occasion mysticaltype experiences having substantial and sustained personal meaning and spiritual significance. Psychopharmacology. 2006;187:268-83.

24. Griffiths RR, Johnson MW, Richards WA, Richards BD, McCann U, Jesse R. Psilocybin occasioned mystical-type experiences: immediate and persisting doserelated effects. Psychopharmacology. 2011;218:649-65.

25. Cameron LP, Benson CJ, Dunlap LE, Olson DE. Effects of N, N-dimethyltryptamine on rat behaviors relevant to anxiety and depression. ACS Chem Neurosci. 2018;9:1582-90.

26. Ly C, Greb AC, Cameron LP, Wong JM, Barragan EV, Wilson PC, et al. Psychedelics promote structural and functional neural plasticity. Cell Rep. 2018;23:3170-82.

27. Vengeliene V, Leonardi-Essmann F, Sommer WH, Marston HM, Spanagel R. Glycine transporter-1 blockade leads to persistently reduced relapse-like alcohol drinking in rats. Biol Psychiatry. 2010;68:704-11.

28. Ezquer F, Quintanilla ME, Morales P, Santapau D, Ezquer M, Kogan MJ, et al. Intranasal delivery of mesenchymal stem cell-derived exosomes reduces oxidative stress and markedly inhibits ethanol consumption and post-deprivation relapse drinking. Addict Biol. 2018;24:994-1007.

29. Sanchis-Segura C, Becker JB. Why we should consider sex (and study sex differences) in addiction research. Addict Biol. 2016;21:995-1006.

30. Vengeliene V, Bachteler D, Danysz W, Spanagel R. The role of the NMDA receptor in alcohol relapse: a pharmacological mapping study using the alcohol deprivation effect. Neuropharmacology. 2005;48:822-9.

31. Hölter SM, Engelmann M, Kirschke C, Liebsch G, Landgraf R, Spanagel R. Longterm ethanol self-administration with repeated ethanol deprivation episodes changes ethanol drinking pattern and increases anxiety-related behaviour during ethanol deprivation in rats. Behav Pharmacol. 1998;9:41-48.

32. Hölter SM, Henniger MSH, Lipkowski AW, Spanagel R. Kappa-opioid receptors and relapse-like drinking in long-term ethanol-experienced rats. Psychopharmacology. 2000;153:93-102.

33. Vengeliene V, Moeller A, Meinhardt MW, Beardsley PM, Sommer WH, Spanagel R, et al. The calpain inhibitor A-705253 attenuates alcohol-seeking and relapse with low side-effect profile. Neuropsychopharmacology. 2016;41:979-88.

34. Cameron LP, Benson CJ, Defelice BC, Fiehn O, Olson DE. Chronic, intermittent microdoses of the psychedelic N, N-dimethyltryptamine (DMT) produce positive effects on mood and anxiety in rodents. ACS Chem Neurosci. 2019;10:3261-70.
35. Davis M, Walters JK. Psilocybin: Biphasic dose-response effects on the acoustic startle reflex in the rat. Pharm Biochem Behav. 1977;6:427-31.

36. Jefsen O, Højgaard K, Christiansen SL, Elfving B, Nutt DJ, Wegener G, et al. Psilocybin lacks antidepressant-like effect in the Flinders Sensitive Line rat. Acta Neuropsychiatr. 2019. https://doi.org/10.1017/neu.2019.15.

37. Koerner J, Appel JB. Psilocybin as a discriminative stimulus: lack of specificity in an animal behavior model for 'hallucinogens'. Psychopharmacology. 1982;76:130-5.

38. Johnstad PG. Powerful substances in tiny amounts: an interview study of psychedelic microdosing. NAD Nord Stud Alcohol Drugs. 2018;35:39-51.

39. Alper K, Dong B, Shah R, Sershen H, Vinod KY. LSD administered as a single dose reduces alcohol consumption in C57BL/6J mice. Front Pharmacol. 2018;9:994.

40. Lea $\mathrm{T}$, Amada $\mathrm{N}$, Jungaberle $\mathrm{H}$. Psychedelic microdosing: a subreddit analysis. J Psychoactive Drugs. 2019. https://doi.org/10.1080/02791072.2019.1683260.

41. Lea $T$, Amada $N$, Jungaberle $H$, Schecke $H$, Klein M. Microdosing psychedelics: motivations, subjective effects and harm reduction. Int J Drug Policy. 2020;75:102600.

42. Horsley RR, Pálenícek T, Kolin J, Valeš K. Psilocin and ketamine microdosing: effects of subchronic intermittent microdoses in the elevated plus-maze in male Wistar rats. Behav Pharmacol. 2018;29:530-6.

43. Spanagel R. Alcoholism: a systems approach from molecular physiology to addictive behavior. Physiol Rev. 2009;89:649-705.

44. Celada P, Victoria Puig M, Casanovas JM, Guillazo G, Artigas F. Control of dorsal raphe serotonergic neurons by the medial prefrontal cortex: involvement of serotonin-1A, GABAA, and glutamate receptors. J Neurosci. 2001;21:9917-29.

45. Puig MV, Celada P, Díaz-Mataix L, Artigas F. In vivo modulation of the activity of pyramidal neurons in the rat medial prefrontal cortex by $5-\mathrm{HT} 2 \mathrm{~A}$ receptors: relationship to thalamocortical afferents. Cereb Cortex. 2003;13:870-82.

46. Vázquez-Borsetti $\mathrm{P}$, Cortés R, Artigas F. Pyramidal neurons in rat prefrontal cortex projecting to ventral tegmental area and dorsal raphe nucleus express $5-\mathrm{HT} 2 \mathrm{~A}$ receptors. Cereb Cortex. 2009;19:1678-86.

47. Vollenweider FX, Vontobel P, Hell D, Leenders KL. 5-HT modulation of dopamine release in basal ganglia in psilocybin-induced psychosis in Man-a PET study with [11C] raclopride. Neuropsychopharmacology. 1999;20:424-33.

48. González-Maeso J, Weisstaub NV, Zhou M, Chan P, Ivic L, Ang R, et al. Hallucinogens recruit specific cortical 5-HT2A receptor-mediated signaling pathways to affect behavior. Neuron. 2007;53:439-52.

49. Poirier R, Cheval H, Mailhes C, Charnay P, Davis S, Laroche S. Paradoxical role of an Egr transcription factor family member, Egr2/Krox20, in learning and memory. Front Behav Neurosci. 2007;1:6.

50. Meinhardt MW, Hansson AC, Perreau-Lenz S, Bauder-Wenz C, Stählin O, Heilig M, et al. Rescue of infralimbic mGluR2 deficit restores control over drug-seeking behavior in alcohol dependence. J Neurosci. 2013;33:2794-806.

51. Smart RG, Storm T, Baker EF, Solursh L. A controlled study of lysergide in the treatment of alcoholism. 1. The effects on drinking behavior. Q J Stud Alcohol. 1966;27:469-82.

52. Meinhardt MW, Sommer WH. Postdependent state in rats as a model for medication development in alcoholism. Addict Biol. 2015;20:1-21.

53. Bell RL, Rodd ZA, Lumeng L, Murphy JM, McBride WJ. The alcohol-preferring P rat and animal models of excessive alcohol drinking. Addict Biol. 2006;11:270-88.

54. Heinz A, Kiefer F, Smolka MN, Endrass T, Beste C, Beck A, et al. Addiction Research Consortium: Losing and regaining control over drug intake (ReCoDe)-from trajectories to mechanisms and interventions. Addict Biol. 2020;25:e12866. 